\title{
Herbivoria floral em Chamaecrista trachycarpa (Vog.) H.S. Irwin \& Barneby, em uma área de campo natural (Telêmaco Borba, PR, Brasil)
}

\author{
Floral herbivory in Chamaecrista trachycarpa (Vog.) H.S. \\ Irwin \& Barneby, in an area of "campo natural" \\ (Telêmaco Borba, PR, Brazil)
}

\author{
Vinicius Messas Cotarelli ${ }^{1}$; Ana Odete Santos Vieira ${ }^{2}$
}

\section{Resumo}

O gênero Chamaecrista Moench está incluído na família Leguminosae, subfamília Caesalpinoideae, tribo Cassieae. Estudos sobre herbivoria e ovoposição de insetos em frutos deste gênero, ou em gêneros da mesma tribo, demonstram interações com insetos da ordem Coleoptera, mais especificamente dos bruquíneos, mas a herbivoria floral é pouco estudada. O objetivo do trabalho foi verificar a presença de herbivoria floral e indicar os potenciais herbívoros de Chamaecrista trachycarpa (Vog.) H.S.Irwin \& Barneby. O experimento foi realizado em uma área de campo natural do Parque Ecológico da Klabin, em Telêmaco Borba, PR, região caracterizada pela transição entre a floresta estacional semidecidual e a ombrófila mista. Foram coletadas três inflorescências, de 15 plantas aleatoriamente, no período da manhã e no da tarde, durante três dias de março de 2008 . Foram coletadas 2156 flores, $38,5 \%$ das quais apresentavam algum sinal de herbivoria. O número total de insetos coletados foi 157 , e a maioria foi coletada no período da tarde. Os coleópteros representaram 146 e os demais foram representados por formigas e formas jovens de hemípteros. A família mais representativa foi Chrysomelidae (Eumolpinae), com 124 insetos coletados, seguido por 22 Curculionidae pertencentes principalmente a subfamília Eumolpinae. Esses podem ser indicados como os principais herbívoros de Chamaecrista.

Palavras-chave: Chamaecrista. Chrysomelidae. Curculionidae. Herbivoria floral. Leguminosae.

\begin{abstract}
The genus Chamaecrista Moench is included in the Leguminosae family, subfamily Caesalpinoideae, tribe Cassieae. Studies on herbivory and oviposition in fruits of this genus, or in genera of the same tribe, demonstrate interactions with insects of the order Coleoptera, more specifically of the bruchines (Chrysomelidae), but there are few records on floral herbivory. The purpose of this work was to verify the presence of floral herbivory and list the herbivorous insects of Chamaecrista trachycarpa (Vog.) $\mathrm{H}$. S. Irwin \& Barneby. The experiment was carried out on a native grassland area of the Ecological Park of Klabin, in Telêmaco Borba, PR, a region characterized by the transition between the semi-deciduous seasonal forest and the mixed ombrophilous forest. Three inflorescences were randomly collected from 15 plants, in the morning and in the afternoon period, during three days in March 2008. Two thousand one hundred and fifty-six (2156) flowers were collected, 38.5\% presenting herbivory. One hundred and fifty-seven (157) insects were collected, most of them in the afternoon period. Coleoptera represented 146 and the others consisted of ants and young forms of hemipteras. The most representative family
\end{abstract}

\footnotetext{
1 Mestrando do Programa de Pós-graduação em Ciências Biológicas da Universidade Estadual de Londrina (UEL), Londrina, PR. E-mail: vinus_bio@yahoo.com.br.

2 Professora do Departamento de Biologia Animal e Vegetal da Universidade Estadual de Londrina (UEL), Londrina, PR.
} 
was the Chrysomelidae (Eumolpinae) with a number of 124, followed by Curculionidae, with a number of 22. Coleoptera, especially the subfamily Eumolpinae, can be indicated as the main Chamaecrista herbivory insects.

Key words: Chamaecrista. Chrysomelidae. Curculionidae. Floral herbivory. Leguminosae.

\section{Introdução}

A subfamília Caesalpinioideae DC. (Leguminosae Juss.) apresenta 2.800 espécies e cerca de 154 gêneros (LEWIS, 2005). Esses gêneros ocorrem nos mais diferentes habitats, com grande variação em suas estruturas reprodutivas e vegetativas. A maioria dos gêneros encontra-se nos trópicos, na África, América e sudeste da Ásia, sendo bem representados no Brasil (RIBEIRO et al., 1999).

O gênero Chamaecrista Moench, que está incluído nesta subfamília (na tribo Cassieae) é composto por aproximadamente 330 espécies distribuídas em seis seções, dos quais 239 são nativas do continente americano (IRWIN; BARNEBY, 1982; LEWIS, 2005).

Muitas espécies deste gênero possuem nectários extraflorais, e neles há glândulas secretoras de néctar não envolvidas diretamente com a polinização. Estudos evolucionários e funcionais destes nectários demonstraram uma relação estreita com a proteção da planta contra herbívoros, e eles podem atrair formigas, cujo papel seria ajudar na defesa contra herbivoria, e também outros insetos, que ocasionalmente visitam as flores (NASCIMENTO; DELL-CLARO, 2007).

Existe um número variado de relações entre insetos e plantas, alguns participam diretamente do processo de polinização, caracterizada pela transferência do pólen (gametófito masculino) para região receptiva da flor feminina (GRIMALDI; ENGEL, 2005), chamados polinizadores; outros podem se alimentar de pólen, diminuindo a quantidade disponível para a polinização, e por isso, são chamados de pilhadores. Alguns se alimentam de partes vegetativas ou reprodutivas das plantas, e são denominados fitófagos (herbívoros) (CORREIA; PINHEIRO; LIMA, 2005; NETO; TEIXEIRA, 2005).

Os insetos estão entre os grupos mais significantes de fitófagos. Em florestas tropicais, são responsáveis por danos entre $10-25 \%$ das partes vegetativas das plantas. Dentro desse grupo, a ordem Coleoptera representa um número significativo de animais fitófagos, pois aproximadamente 35\% (125.000) das espécies são consideradas fitófagas (GRIMALDI; ENGEL, 2005).

Dentro da ordem podem ser citadas três famílias de besouros fitófagos: Cerambycidae, que compreende 35.000 espécies, cujos adultos se alimentam de folhas, brotos e pólen e as larvas se desenvolvem no floema; Chrysomelidae, com cerca de 38.000 espécies, que inclui a maioria de insetos que se alimentam das partes externas da planta e as larvas se desenvolvem exclusivamente em frutos e sementes; e Curculionidae com 44.000 espécies, com adultos se alimentando em geral de folhas, brotos e muitas vezes de pólen (GRIMALDI; ENGEL, 2005).

Alguns estudos sobre herbivoria e ovoposição de insetos em frutos de plantas de Chamaecrista, ou mesmo em gêneros da mesma tribo, foram realizados recentemente, apontando uma estreita relação com insetos da ordem Coleoptera, mais especificamente de bruquíneos (Chrysomelidae) (MADEIRA; FERNANDES, 1999; MADEIRA; RIBEIRO; FERNANDES, 1998; RUHREN, 2003; SARI; RIBEIRO-COSTA; ROPER, 2005; SILVA; RIBEIRO-COSTA; JOHNSON, 2003).

Em relação à herbivoria floral em Chamaecrista, pouco foi relatado, só a presença de algumas 
espécies de coleópteros Burpestidae (Agrilus sp., Tetragonoschema sp.) que se alimentam nas pétalas ou copulam nas flores (NASCIMENTO; DELLCLARO, 2007).

Para algumas espécies vegetais, altas taxas de predação floral podem resultar em uma menor taxa de produção de sementes e conseqüentemente de descendentes (LEAVITT; ROBERTSON, 2006; NASCIMENTO; DELL-CLARO, 2007; RUHREN, 2003). Segundo Mccall e Irwin (2006), flores predadas podem ser menos visitadas por polinizadores, por causa da alteração da quantidade e qualidade de caracteres florais importantes para interação com estas espécies.

Abelhas, as principais espécies relacionadas à polinização do gênero Chamecrista, por exemplo, tendem a evitar flores que apresentam pétalas e/ou estames com danos (KRUPNIC; WEIS; CAMPBELL, 1999).

A partir disto levantam-se as seguintes perguntas: existe herbivoria floral em Chamaecrista trachycarpa? Quais são os herbívoros florais desta espécie? Portanto, o objetivo do trabalho foi o de verificar a presença de herbivoria floral e indicar os herbívoros.

\section{Material e métodos}

Área de estudo - O experimento foi desenvolvido na fazenda Monte Alegre, pertencente à empresa Klabin S.A., e situada no município de Telêmaco Borba, PR, localizado na região central do Estado, nas coordenadas geográficas $24^{\circ} 12^{\prime} 42^{\prime \prime} \mathrm{S}$ e $50^{\circ}$ $33^{\prime} 26$ " W, com uma altitude média de $885 \mathrm{~m}$ (AZEVEDO et al., 2008).

A fazenda está localizada na região do médio curso do rio Tibagi, ocupa uma área de 126 mil hectares, dos quais 46.000 ha são de áreas nativas. O Parque Ecológico, presente na fazenda Monte Alegre, foi implantado em 1980, possui uma área total de 11.196 ha, dos quais 7.883 ha são cobertos por florestas naturais, algumas dessas ainda em estado primitivo ou pouco alteradas (AZEVEDO et al., 2008) (Figura 1).

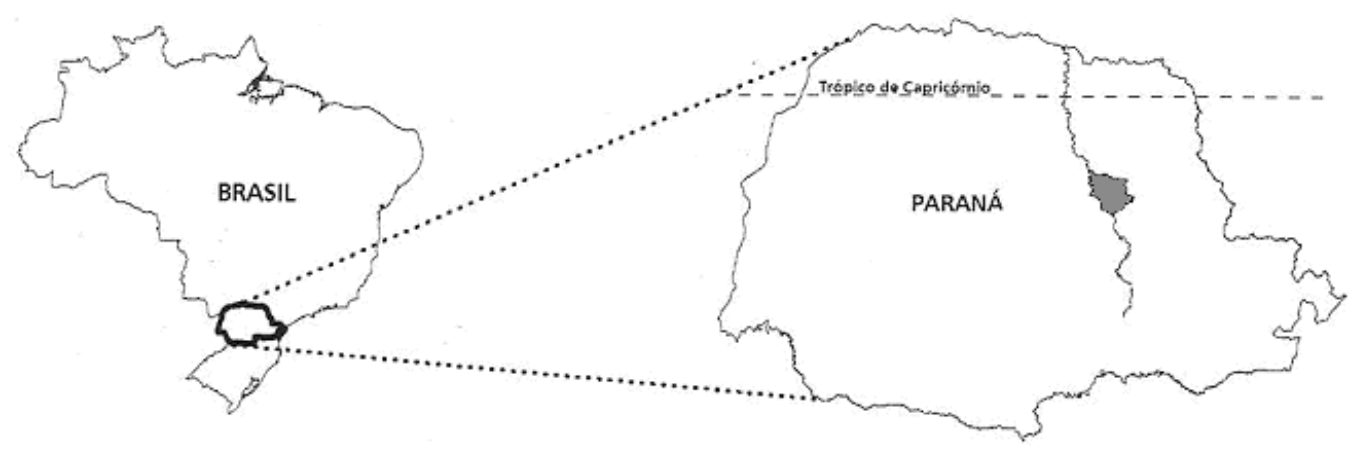

Figura 1. Localização da fazenda Monte Alegre no estado do Paraná (seta), onde está localizado o Parque Ecológico da Klabin.

Fonte: Adaptado de Azevedo et al., 2008. 
Segundo Köppen, o clima predominante na região é do tipo subtropical transicional para o temperado propriamente dito, úmido, mesotérmico, sem estação seca definida $(\mathrm{Cfa} / \mathrm{Cfb})$. Os verões são quentes e chuvosos e os meses com precipitações relativamente menores são Abril, Julho e Agosto e as geadas ocorrem com pouca freqüência. A precipitação pluviométrica anual média é de $1.490 \mathrm{~mm}$. A temperatura anual média varia de $15,6^{\circ} \mathrm{C}$ a $22,2^{\circ} \mathrm{C}$, com um valor médio anual de $19,3^{\circ} \mathrm{C}$. A umidade relativa do ar apresenta uma média anual de 77\% (dados da Estação Meteorológica da Lagoa (1947-2004) e AZEVEDO et al., 2008).

Podem ser encontrados três tipos de formações vegetais no Parque Ecológico: floresta estacional semidecidual, floresta ombrófila mista e mancha de campos naturais, além de ecótonos formados entre as mesmas (AZEVEDO et al., 2008).

Espécie - A espécie estudada pertence ao gênero Chamaecrista Moench., à família Leguminosae Juss, subfamília Caesalpinoideae DC., tribo Cassieae Bronn, seção Absus. Possui porte arbustivo, freqüentemente presente em vegetações do tipo campos naturais, cerrados ou borda de matas. Possui inflorescências racemosas, terminais, flores vistosas com cálice e corola pentâmeros, pétalas amarelas. Espécies deste gênero apresentam uma antese diurna, que ocorre nas primeiras horas da manhã, e geralmente disponibilizando o pólen por toda manhã (IRWIN; BARNEBY, 1982; NASCIMENTO; DELL-CLARO, 2007).

Metodologia - $\mathrm{O}$ experimento foi desenvolvido em uma área de campo natural antropizado, localizada dentro do Parque Ecológico da Klabin, em frente ao Centro de Interpretação da Natureza "Franz Krajcberg". Esta área foi escolhida por possuir populações de plantas de Chamaecrista trachycarpa.

$\mathrm{Na}$ área, foram coletadas inflorescências de 15 plantas aleatoriamente, em dois períodos do dia, de manhã a partir das 06h30min, e à tarde das 17h00min totalizando 30 plantas por dia, nos dias 12, 13 e 14 de março de 2008. As coletas tiveram a duração de aproximadamente uma hora.

Em cada planta, foram coletadas três inflorescências, totalizando 270 no final do experimento. As inflorescências foram coletadas em sacos de papel de $10,5 \times 25 \mathrm{~cm}$, e, posteriormente, guardadas no freezer por aproximadamente $12 \mathrm{~h}$. Quando retiradas, contou-se o número de insetos presentes nas inflorescências, o de flores, o de botões e o de flores com algum tipo de predação. Os insetos retirados foram colocados em um frasco com álcool 70\% para posterior identificação e depósito no Museu de Zoologia da Universidade Estadual de Londrina.

Análise dos dados - Para a compilação dos dados, foi usado o programa Excel 2000, por meio do qual foram retiradas as médias de todos os dados por período de coleta (manhã e tarde) e calculado o desvio padrão. Foi utilizado o programa Biostat 4.0 para aplicar o teste de correlação linear de Pearson entre a média do número de flores predadas e média de insetos encontrados em cada período.

\section{Resultados}

Foi analisado um total de 2156 flores, em 270 inflorescências, ao longo do período de

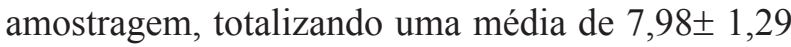
flores por inflorescências. Flores com algum sinal de herbivoria totalizaram $38,5 \%$.

A média de flores predadas por inflorescência, no período da manhã, foi de $6,18 \pm 0,6$, a de flores totais foi de 20,36 $\pm 1,23$, e de botões, 17,22 $\pm 2,12$. No período da tarde as flores predadas representaram $12,29 \pm 1,7$, as flores totais, $27,56 \pm 2,03$, e os botões, $24,71 \pm 1,81$.

O número total de insetos coletados foi 157 , e a maioria foi coletada no período da tarde (117). Os coleópteros representaram um total de $146(92,9 \%)$, o restante $(8,1 \%)$ foi representado por formigas e formas jovens de hemípteros. A família mais representativa foi Chrysomelidae (Eumolpinae), 
com 124 (80,5\%), seguido por Curculionidae 22 (14,5\%) (Tabela 1).

Nos dois períodos, uma forte correlação positiva foi calculada entre o número de insetos coletados e o número de flores com algum tipo de dano, pois para ambos os períodos $\mathrm{r}$ (Pearson) $=0,9945$. (Figura 2).

Tabela 1. Número de indivíduos coletados nas inflorescências de Chamaecrista trachycarpa por família de coleóptero em cada período do dia.

\begin{tabular}{lcccc}
\hline & $\begin{array}{c}\text { Número de indivíduos de } \\
\text { Coleoptera - Chrysomelidae } \\
\text { (Eumolpinae) }\end{array}$ & $\begin{array}{c}\text { Número de indivíduos de } \\
\text { Coleoptera - Curculionidae }\end{array}$ & $\begin{array}{c}\text { Número de } \\
\text { indivíduos de } \\
\text { outros insetos }\end{array}$ & $\begin{array}{c}\text { Total de } \\
\text { individuos por } \\
\text { período (\%) }\end{array}$ \\
\hline Manhã & $32(20,7 \%)$ & $7(4,6 \%)$ & $5(3,2 \%)$ & $44(28,5 \%)$ \\
Tarde & $92(59,8 \%)$ & $15(9,9 \%)$ & $3(1,8 \%)$ & $110(71,5 \%)$ \\
Total (\%) & $124(80,5 \%)$ & $22(14,5 \%)$ & $8(5 \%)$ & \\
\hline
\end{tabular}

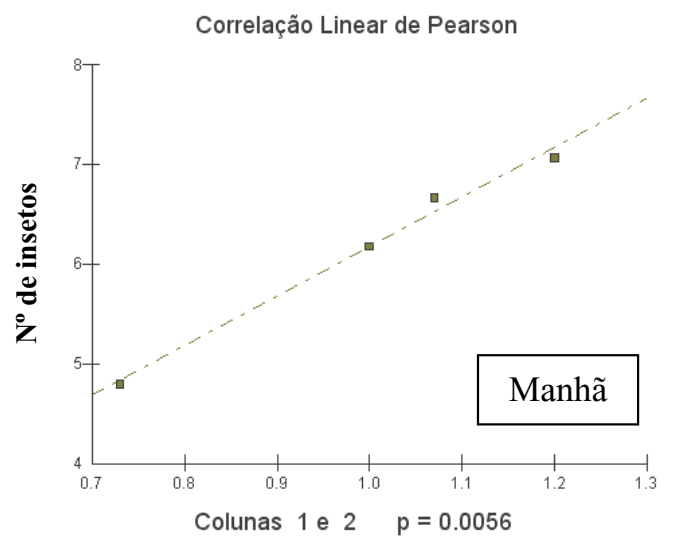

$\mathbf{N}^{\circ}$ de flores predadas

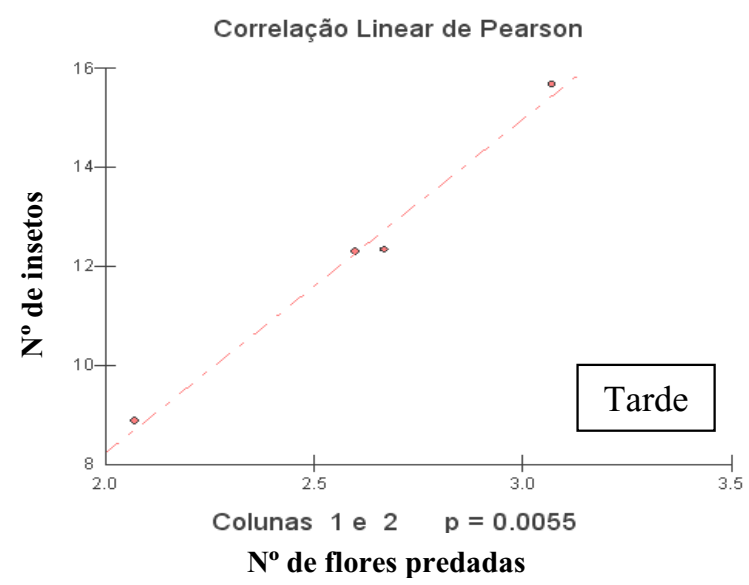

Figura 2. Correlação linear de Pearson entre o número de insetos e o de flores predadas. 


\section{Discussão}

A maioria dos insetos coletados pertence à ordem Coleoptera. Não se pode afirmar que eles participam de uma forma direta da polinização, pois este grupo de inseto poliniza plantas dotadas de outras síndromes principais (JONES; LITLE, 1983; RAVEN; EVERT; EICHHORN, 2007; SOUTHGATE, 1979). Nascimento e Dell-Claro (2007), estudando uma espécie de Chamaecrista, em área de cerrado em Minas Gerais, relacionaram entre os visitantes florais mais freqüentes, alguns himenópteros, a maioria Apidae, que podem ser listados como polinizadores efetivos, pelo fato de a vibração das asas destes insetos liberar o pólen das anteras poricidas e transferi-lo para o estigma. $\mathrm{Na}$ maioria das espécies do gênero Chamaecrista é encontrada a antese diurna (NASCIMENTO; DELL-CLARO, 2007), e, neste experimento, o maior número de botões florais presentes no período da tarde, reforça a abertura da flor durante o dia e a fraca interação dos coleópteros com a polinização. Estes autores também listaram alguns coleópteros (Brupestidae), que foram relacionadoss à herbivoria floral da espécie.

O maior número de flores predadas e de coleópteros coletados no período da tarde pode ter uma relação direta com a sua atividade ao longo do dia, pois os picos de atividade ocorrem em temperaturas mais elevadas (SOUTHGATE, 1979).

A correlação Linear de Pearson, gerada entre os números de insetos e flores predadas, mostra uma forte correlação positiva entre as duas variáveis analisadas, sugerindo que estas subfamílias têm grande probabilidade de serem os herbívoros florais nesta espécie.

A família Chrysomelidae é a mais numerosa encontrada no experimento, e acerca dela há um maior número de trabalhos relacionados diretamente com espécies do gênero Chamaecrista e outros gêneros da mesma tribo, como Senna e Cassia (MADEIRA; RIBEIRO; FERNANDES, 1998; RIBEIRO-COSTA; COSTA， 2002; RUHREN,
2003; SARI; RIBEIRO-COSTA; ROPER, 2005; SILVA; RIBEIRO-COSTA; JOHNSON, 2003). Johnson e Romero (2004) citam que espécies da família Chrysomelidae, alimentam-se de partes da planta, como folhas, brotos, raízes e flores.

Larvas de uma das subfamílias, Bruchinae, não encontradas neste experimento, alimentam-se de sementes de plantas, pois estas se desenvolvem nos frutos, tendo como principais hospedeiros espécies da família Leguminosae (SOUTHGATE, 1979). Por exemplo, Sári, Ribeiro-Costa e Roper (2005) citam a ovoposição de espécies de Sennius (Bruchinae Chrysomelidae) em vagens imaturas de uma espécie da tribo Cassineae.

A subfamília, Eulmopinae (Chrysomelidae), contém o maior número de espécies fitófagas do mundo. A maioria de suas espécies está associada diretamente com a predação foliar quando adultos e, quando em fase larval, nas raízes ou mesmo nas folhas (XING-PENG; CHENG DE, 2007). Entretanto, no experimento foi a mais numerosa encontrada dentro das flores coletadas no período da tarde, diferindo da literatura onde na grande maioria dos casos é considerada simplesmente predadora foliar.

A literatura não menciona nenhuma relação direta de espécies do gênero Chamaecrista e os eumolpíneos, mas há relatos de interações com indivíduos de Acacia mearnsii De Wild. (Leguminosae-Mimosoideae) em plantios comerciais no sudeste da Austrália, relacionados diretamente com a herbivoria foliar (HUNT; GULLAN; REID, 1996). Ainda dentro da família Leguminosae, Cabrera, Lazaro e Nasca (2000) citam a herbivoria foliar de uma espécie de eumolpíneo em uma área de cultivo de soja, na região de Tucuman, Argentina.

No Brasil existem poucos relatos de herbivoria por eumolpíneos mas, são conhecidas suas notórias associações com plantações de cacau (Theobroma cacao L.) na região sul da Bahia (FERRONATO, 2000), e eles são considerados como pragas desta cultura. 
A presença dos eumolpíneos nesta região do estado do Paraná pode estar ligada a grande área de plantios comerciais de Eucalyptus ao redor do Parque Ecológico da Klabin, pois, em algumas regiões da Austrália, eles são considerados praga para estes plantios comerciais (LOCH, 2005, 2006), e podem estar utilizando as espécies de Chamaecrista para a reprodução e fitofagía.

A outra família registrada, Curculionidae, não apresentou uma relação muito estreita com as espécies da família Leguminosae, como ocorre com os Chrysomelidae. Schlindwein e Martins (2000) citam que Prestimeris sp. (Curculionidae) visitaram as flores de uma espécie de Malvaceae, em geral, para se alimentarem de pólen e ainda as utilizavam como locais para passar a noite, ou somente para descanso. Volovnick (1996) relatou para Linux sp. (Curculionidae) um ciclo de vida ligado diretamente a espécies de Brassicaceae, e nele adultos se alimentavam das folhas da espécie e colocavam os ovos nos estames, mostrando as diferentes relações que podem ocorrer desta família de insetos com espécies vegetais.

As duas famílias de coleópteros relatadas no experimento podem ser herbívoras florais da C. trachycarpa, e estão relacionadas somente à herbivoria, associada à ovoposição ou simplesmente utilizando as mesmas para o descanso. Os Chrysomelidae - Eumolpinae encontrados em maior número podem ser listados como principais herbívoros florais da espécie nesta época do ano, na região do estudo.

\section{Agradecimentos}

Dr. Carlos Eduardo de Alvarenga Julio, professor do Departamento de Biologia Animal e Vegetal da Universidade Estadual de Londrina (UEL), pela identificação das famílias e subfamílias das espécies da ordem Coleoptera. A Universidade Estadual de Londrina pelo transporte e materiais fornecidos, ao Parque Ecológico da Klabin, pela concessão do alojamento e alimentação fornecida durante a disciplina Biologia de Campo do curso de Mestrado em Ciências Biológicas, período em que o trabalho foi realizado.

\section{Referências}

AZEVEDO, T. I. N.; SEKIAMA, M. L.; VIEIRA, A. O. S; BENNEMANN, S. T. Descrição física da micro bacia do Ribeirão Varanal e caracterização dos trechos. In: BENNEMANN, S. T.; SHIBATTA, O. A.; VIEIRA, A. O. S. (Org.). A flora e a fauna do Ribeirão Varanal, um estudo da biodiversidade no Paraná. Londrina: Eduel, 2008. p. 5-14.

CABRERA, N.; LAZARO, H.; NASCA, A. Caracterización morfológica y presencia de Maecolaspis monrosi Bechyné (Coleoptera: Chrysomelidae: Eumolpinae) en el cultivo de soja en Tucuman. Anais da Sociedade Entomológica do Brasil, Londrina, v. 29, n. 2, p. $277-284,2000$.

CORREIA, M. C. R.; PINHEIRO, M. C. B.; LIMA, H. A. Biologia floral e polinização de Arrabidadea conjugata (Vell.) Mart. (Bignoniaceae). Acta Botanica Brasílica, São Paulo, v. 19, n. 3, p. 501-510, 2005.

FERRONATO, E. M. O. A cápsula da espermateca dos Eumolpinae (Coleoptera: Chrysomelidae) associados a Theoboma cacau L. (Sterculiaceae) do sul da Bahia. Anais da Sociedade Entomológica do Brasil, Londrina, v. 29, n. 1, p. 57-63, 2000.

GRIMALDI, D.; ENGEL, M. S. Evolution of the insects. United Kingdom: Cambridge University Press, 2005.

HUNT, A. J.; GULLAN, P. J.; REID, C. A. M. Chrysomelidae (Coleoptera) and other phytophagous insects of Black Watlle, Acacia mearnsii De Wild., in southeastern Australia. Australian Journal of Entomology, Canberra, v. 35, n. 1, p. 85-92, 1996.

IRWIN, H. S.; BARNEBY, R. C. The Americam Cassinae: a synoptical revision of the leguminosae tribe Cassiaea subtribe Cassiinae in the New World. Memoirs of The New York Botanical Garden, New York, v. 35, n. 2, p. 637-895, 1982.

JOHNSON, C. D.; ROMERO, J. A review of evolution of oviposition guilds in the Bruchidae (Coleoptera). Revista Brasileira de Entomologia, Curitiba, v. 48, n. 3, p. 401408, 2004.

JONES, C. E.; LITLE, R. J. Handbook of experimental pollination biology. New York: Scientific and Academic, 1983. 
KRUPNIC, G. A.; WEIS, A. E.; CAMPBELL, D. R. The consequences of floral herbivory for pollinator service to Isomeris Arborea. Ecology, New York, v. 80, n. 1, p. 125134, 1999.

LEAVITT, H.; ROBERTSON, I. P. Herbivory by chrysomelid beetles (Phylotrea sp.) is detrimental to pollination and seed production in Lepidium papilliferum (Brassicaceae).Ecological Entomology, United Kingdom, v.31, n. 6, p. 657-660, 2006.

LEWIS, G. P. Tribe Cassieae. In: LEWIS, G. P.; SCHRIRE, B; MACKINDER, B.; LOCK, M. (Ed.). Legumes of the world. Richmond: The Royal Botanic Gardens, 2005. p. 122.

LOCH, A. D. Mortality and recovery of eucalypt beetle pest and beneficial arthropod populations after commercial applications of alpha-cypermethrin. Forest Ecology and Manegement, New York, v. 217, n. 2-3, p. 255-265, Oct. 2005.

LOCH, A. D. Phenology of Eucaliptus weevil, Gonipterus scutellatus Gyllenhal (Coleoptera: Curcullionidae), and chrysomelid beetles, in Eucalyptus globules plantations in south-western Australia. Agricultural and Forest Entomology, United Kingdom, v. 8, n. 2, p. 155-165, 2006.

MADEIRA, J. A.; FERNANDES, G. W. Reproductive phenology of sympatric taxa of Chamaecrista (Leguminosae) in Serra do Cipó, Brazil. Journal of Tropical Ecology, United Kingdom, v. 15, n. 44, p. 463479, 1999.

MADEIRA, J. A.; RIBEIRO, K. T.; FERNANDES, G. W. Herbivory, tanins and sclerophylly in Chamaecrista linearifolia (Fabaceae) along an altitudinal gradient. Brazilian Journal of Ecology, Rio Claro, v. 2, n. 1, p. 1-10, 1998.

McCALL, A. C.; IRWIN, R. E. Florivory: the intersection of pollination and herbivory. Ecology Letters, Montpellier, v. 9, n. 12, p. 1351-1365, 2006.

NASCIMENTO, E. A.; DELL-CLARO, K. Floral visitors of Chamaecrista debilis (Vogel) H.S.Irwin \& Barneby (Fabaceae-Caesalpinoideae) at Cerrado of estação ecológica do jataí, São Paulo State, Brazil. Neotropical Entomology, Piracicaba, v. 36, n. 4, p. 619-624, 2007.

NETO, H. F. P.; TEIXEIRA, R. C. Florivory and sex ratio in Annona dioica St. Hil.(Annonaceae) in the Pantanal at Nhêcolandia, southwestern Brazil. Acta Botanica Brasílica, São Paulo, v. 20, n. 2, p. 405-409, 2005.
RAVEN, P. H.; EVERT, R.; EICHHORN, S. E. Biologia vegetal. 7. ed. Rio de Janeiro: Guanabara Koogan, 2007. p. $470-492$.

RIBEIRO, J. E. L. S.; HOPKINS, M. J. G.; VICENTINI, A.; SOTHERS, C. A.; COSTA, M. A. S.; BRITO, J. M.; SOUZA, M. A. D.; MARTINS, L. H.; LOHMANN, L. G.; ASSUNÇÃO, P. A. C. L.; PEREIRA, E. C.; SILVA, C. F.; MESQUITA, M. R.; PROCÓPIO, L. C. Flora da reserva Duke: guia de identificação das plantas vasculares de uma floresta de terra firme na Amazônia central. Manaus: INPA, 1999.

RIBEIRO-COSTA, C. S.; COSTA, A. S. Comportamento e ovoposição de Bruchidae (Coleoptera) predadores de sementes de Cassia leptophylla (Vogel) (Caesalpinaceae), morfologia dos ovos e descrição de uma nova espécie. Revista Brasileira de Zoologia, São Paulo, v. 19, Supl. 1, p. 305-316, 2002.

RUHREN, S. Seed predators are undeterred by nectarfeeding ants on Chamaecrista (Caesalpinaceae). Plant Ecology, Dordrecht, v. 166, n. 2, p. 189-198, 2003.

SARI, L. T.; RIBEIRO-COSTA, C. S.; ROPER, J. J. Dinâmica de bruquíneos (Coleoptera, Chrysomelidae) em Senna multijuga (Rich.) H. S.Irwin \& Barneby (Caesalpinaceae). Revista Brasileira de Zoologia, São Paulo, v. 22, n. 1, p. 169-174, 2005.

SCHLINDWEIN, C.; MARTINS, C. F. Competition between the oligolectic bee Ptilothrix plumata (Anthophoridae) end the flower closing beetle Pristimerus calcaratus (Curculionidae) for floral resources of Pavonia cancellata (Malvaceae). Plant Systematics Evolution, New York, v. 224, n. 3-4, p. 183-194, 2000.

SILVA, J. A. P.; RIBEIRO-COSTA, C. S.; JOHNSON, C. D. Sennius Bridwell (Coleoptera, Bruchidae): novas espécies predadoras de semente de Chamaecrista Moench (Caesalpinaceae) da Serra do Cipó, Santana do Riacho, Minas Gerais, Brasil. Revista Brasileira de Zoologia, São Paulo, v. 20, n. 2, p. 269-277, 2003.

SOUTHGATE, B. J. Biology of the Bruchidae. Annual Reviews of Entomology, Palo Alto, v. 24, n. 1, p. 449473, 1979.

VOLOVNICK, S. V. On biology of Lixus albomarginatus (Col., Curculionidae). Anzeiger fur Schidlingskde Pflanzenschutz, Berlin, v. 69, n. 2, p. 40, 1996.

XING-PENG, L. I.; CHENG DE, L. I. Check list of Eumolpinae (Coleoptera, Chrysomelidae) from Mao'ershan region including one new record species from China. Journal of Forestry Research, Harbin, v. 18, n. 1, p. $65-68,2007$. 